\title{
Manga Y Su Incidencia En La Comprensión Lectora Del Idioma Inglés En Estudiantes De Educación Superior
}

\section{Vallejo Barreno Carina Fernanda}

Magister en Lingüística y Didáctica de la Enseñanza de Idiomas Extranjeros Docente del Centro de Idiomas, Escuela Superior Politécnica de Chimborazo

\section{Castillo Mazón Holger Patricio}

Licenciado en Ciencias de la Educación Profesor de Idiomas Inglés Docente del Centro de Idiomas, Escuela Superior Politécnica de Chimborazo

\section{Haro Carrillo Danny Omar}

Licenciado en Ciencias de la Educación Profesor de Idiomas Inglés

Unidad Educativa "Juan de Velasco"

\section{Guerrero Chacón Keila Esther}

Magister Scientiarum en Supervisión Educativa

Docente del Centro de Idiomas, Escuela Superior Politécnica de Chimborazo

\section{Resumen}

La investigación tuvo como propósito analizar la incidencia del Manga en la comprensión lectora del idioma inglés en los estudiantes del tercer nivel del Centro de Idiomas de la Escuela Politécnica de Chimborazo en el periodo académico septiembre 2018 febrero 2019. Se consideró como variable independiente el Manga y como variable dependiente la comprensión lectora del idioma inglés. La investigación tuvo un enfoque cuantitativo, un diseño cuasi experimental, hipotético deductivo, y fue de tipo descriptiva, correlacional, de campo y bibliográfica. Se utilizó un plan de intervención que sirvió como estrategia elaborada con un set de historietas japonesas tales como: Harem, Magical Girlfriend, Josei, Seinen, Mecha. Del mismo modo, se realizaron evaluaciones basadas en el examen internacional Preliminary English Test (PET) de Cambridge con el fin de valorar la variable dependiente. Las evaluaciones fueron realizadas a dos grupos: de control (20 estudiantes - sin utilizar mangas) y el experimental (18 estudiantes - aplicando mangas con un plan de intervención). Las evaluaciones fueron desarrolladas en dos momentos (pre y post test) y los resultados obtenidos fueron tabulados, analizados, interpretados y sometidos a la prueba estadística $Z$, donde el hallazgo más significativo fue, que el 
Manga incidió positivamente en la comprensión lectora en inglés de los estudiantes del grupo experimental.

Palabras claves: Manga, Incidencia, Compresión lectora, Inglés, historietas

\section{Sleeve and its Incidence in Reading and the Understanding of English Language in Higher Education Students}

\section{Vallejo Barreno Carina Fernanda}

Magister en Lingüística y Didáctica de la Enseñanza de Idiomas Extranjeros Docente del Centro de Idiomas, Escuela Superior Politécnica de Chimborazo

\section{Castillo Mazón Holger Patricio}

Licenciado en Ciencias de la Educación Profesor de Idiomas Inglés Docente del Centro de Idiomas, Escuela Superior Politécnica de Chimborazo

\section{Haro Carrillo Danny Omar}

Licenciado en Ciencias de la Educación Profesor de Idiomas Inglés

Unidad Educativa "Juan de Velasco"

\section{Guerrero Chacón Keila Esther}

Magister Scientiarum en Supervisión Educativa

Docente del Centro de Idiomas, Escuela Superior Politécnica de Chimborazo

\section{Abstract}

This paper focuses on analyzing the incidence of Manga in English language reading comprehension in the third level students of the Language Center at Escuela Politécnica de Chimborazo in the academic period September 2018 - February 2019. The independent variable was Manga, while the dependent variable was English language reading comprehension. The research had a quantitative approach, quasi-experimental design, hypothetical deductive, descriptive and correlational field, and bibliography. An intervention plan was used and it served as a strategy with a set of Japanese comic strips such as: Harem, Magical Girlfriend, Josei, Seinen, and Mecha. Moreover, evaluations based on the international examination Preliminary English Test (PET) of Cambridge were carried out. This was done in order to assess the dependent variable. The evaluations were made in two groups: control (20 students - without Mangas) and the experimental (18 students - applying Mangas with an intervention plan). The evaluations were 
developed in two moments (pre and posttest) and the results obtained were tabulated, analyzed, interpreted, and subjected to the $\mathrm{Z}$ statistical test. Here, the most significant finding shows that Manga had a positive impact on the English reading comprehension of the experimental group.

Keywords: Manga, Incidence, Reading Comprehension, English, Comics

\section{INTRODUCCIÓN}

Al revisar varias bibliotecas virtuales, se pudo verificar que existen investigaciones relacionadas a la presente investigación; entre ellas tenemos:

- Sara Paola Lengua Salinas en el año del 2015, realiza la investigación "EL CÓMIC: UNA OPORTUNIDAD DE MOTIVACIÓN Y APRENDIZAJE EN LAS CLASES DE INGLÉS COMO L2, en la Pontificia Universidad Javeriana de Bogotá, investigación en la que se planteó como objetivo evaluar el impacto del uso del cómic como herramienta motivadora y potenciadora del proceso de aprendizaje a través del diseño de estrategias de intervención pedagógica en la clase de inglés. En ella se llegó a concluir que el uso y creación de un cómic tiene un efecto motivador que permitió a los estudiantes avanzar en su aprendizaje mientras expresaban sus intereses y gustos.

- Isabel García Martínez en el año del 2013 desarrolla la investigación "EL CÓMIC COMO RECURSO DIDÁCTICO EN EL AULA DE LENGUAS EXTRANJERAS" en la Universidad de CantabriaEspaña, la misma que aportó a fomentar el uso del cómic como una herramienta didáctica complementaria en el aula de lenguas extranjeras, es decir, utilizar el comic para efectos motivadores que mejoren el hábito lector, favorezcan el aprendizaje de la comprensión lectora y enriquezcan el vocabulario.

- Mónica Jacqueline Tipán Alquinga en la Universidad Central del Ecuador en el año 2017 realizó la investigación "EL CÓMIC COMO ESTRATEGIA DE ATENCIÓN PARA LOS ESTUDIANTES DE INGLÉS" donde se plantea "¿De qué manera influye el comic como estrategia para lograr la atención de los estudiantes de inglés?”. En la investigación se concluye que existe un escaso uso del comic como estrategia en las clases de inglés; sin embargo, existe motivación hacia el uso del recurso por los estudiantes que leen cómics y es recomendable que se utilice para mejorar el nivel de comprensión y se incentive la criticidad literal o inferencial.

- Diego Mauricio Delgadillo Pérez en el año 2016, desarrolló la tesis titulada "EL CÓMIC: UN RECURSO DIDÁCTICO PARA FOMENTAR LA LECTURA CRÍTICA" en la Universidad Pedagógica Nacional Central de Bogotá-Colombia, en la que se 
demuestra que la lectura constante de cómics ayuda a los estudiantes a ser críticos. En dicha tesis se concluye que emplear el cómic como recurso didáctico contribuye activamente en los procesos de lectura crítica, ya que al relacionar la imagen con el texto los estudiantes son capaces de asociar dichos elementos para emitir juicios críticos o valorativos.

- Joan Antoni Pomata García en la Universidad de Alicante en España en el año 2011-2012, realiza la investigación "EL MANGA COMO RECURSO DE ENSEÑANZA, ADQUISICIÓN Y APRENDIZAJE EN LA CLASE DE ESPAÑOL COMO LE/L1/L2", donde se concluye que el Manga es útil en la enseñanza del idioma español debido a los beneficios que aporta en el aprendizaje de un nuevo idioma. Entre otras cosas se menciona que el Manga posee un potencial para el aula de ELE, EL1 y EL2 ya que las actividades que se pueden elaborar a través del Manga pueden variar según la dificultad que necesite el alumno. Además, el Manga se puede adaptar a las cuatro destrezas lingüísticas.

- Vicente Javier Manjarrez Vásquez, estudiante de la Universidad Técnica De Ambato en el año 2015, realiza una investigación para lograr, "LA IMPLEMENTACIÓN DE CÓMICS DIGITALES", con el objetivo de alcanzar el desarrollo de la destreza de lectura en el idioma inglés en los estudiantes de nivel intermedio alto B2 y avanzado $\mathrm{C} 1$ de los cursos abiertos que se dictan en la Universidad Técnica de Ambato. De acuerdo a Manjarrez 2015, el cómic no es solo para público infantil; el cómic es un amplio mundo en el que están inmersos lectores de nivel básico, intermedio y avanzado.

Con base en las investigaciones anteriores se puede indicar que la comprensión lectora, entendiéndola como destreza receptiva, es uno de los desafíos que docentes y estudiantes enfrentan a diario. Este fenómeno es reconocido mundialmente y es evidenciado en los resultados de la prueba estandarizada PISA (evaluación en español). En el caso del idioma inglés, en la búsqueda de la información, no se ha evidenciado que exista una evaluación para América Latina que mida el nivel de comprensión lectora.

Ortega (1998), afirma que, si los estudiantes eran capaces de denominar las palabras, la comprensión, por ende, sería automática. Sin embargo, a medida que los docentes guiaban más su actividad a la decodificación, fueron comprobando que la mayoría de los estudiantes no entendían lo que leían. Es decir que devela con claridad que existen problemas de comprensión lectora.

Castillo (2013), sostiene que "En una encuesta del Instituto Nacional de Estadísticas y Censos (INEC) realizada en cinco ciudades del Ecuador, $27 \%$ de las personas entrevistadas declaró no tener hábito de leer. 
De ellas, 56,8\% dijo que no le interesaba la lectura". Lo anterior demuestra que en Ecuador las personas tienen pocos hábitos de lectura en español y consecuentemente en la lectura en inglés, entendiendo que a nivel de educación básica y de bachillerato no se logra desarrollar la comprensión lectora y consecuentemente esto afecta a la educación superior.

Vega (2012), afirma que "En el mercado editorial del Japón el Manga es considerado como una de las tradiciones más influyentes en el mundo ya que incluye todos los géneros y está dirigida a todo público". El mismo autor sostiene que "las historietas Manga pueden ser encontradas en varios formatos, así como en dibujos animados o animes, videojuegos, películas, novelas o imágenes reales". Debido a su diversidad de temas y géneros, es evidente que su popularidad se incrementa constantemente.

Flores (2010) sostiene igualmente que las historietas son consideradas como un recurso didáctico de importancia para desarrollar nuevas estrategias, como incluir el humor dentro de la pedagogía, lo que implica métodos, técnicas y recursos como eje central. Esto quiere decir que se podría proporcionar a los estudiantes una metodología para perfeccionar el desenvolvimiento en una lengua extranjera incidiendo en las destrezas lectoras, escritas u orales.

Garrido (2010) afirma que "La historieta contiene cualidades lingüísticas que permiten alcanzar un aprendizaje significativo debido a los procesos y técnicas que esta posee". Por lo tanto, utilizar nuevos métodos y estrategias por parte de los docentes incidiría en el aprendizaje significativo en el proceso de enseñanza aprendizaje,

Al trabajar con Mangas el docente debe seleccionarlos de acuerdo al nivel, edad y contenidos del micro currículo. Entre los aportes de Mangas se tiene:

1. Promueve la creatividad de los alumnos para la resolución de problemas de la vida diaria.

2. Genera hábitos de lectura.

3. Permite la identificación espaciotemporal.

4. Apoya al aprendizaje de arte y estética.

5. Despierta el interés de una nueva cultura, en este caso la cultura japonesa promotora del Manga.

Abal (2010), indica que los beneficios del Manga son múltiples. Entre ellos destaca la imaginación, creatividad, comunicación, cohesión grupal y sin duda la integración en diferentes niveles como básico, intermedio o avanzado, permitiendo así mejorar su capacidad lectora $\mathrm{y}$ crítica.

El Manga en la educación es considerado como un recurso educativo, pues su metodología permite mejorar las destrezas lectoras. Por 
tal motivo es un material didáctico totalmente aceptable y divertido para el fortalecimiento del aprendizaje.

Basensi (2010), menciona que la historieta influye positivamente en la educación pues "es un recurso motivador, su desarrollo secuencial es idóneo para la iniciación en el género narrativo, supone un medio de comunicación que permite comprender procesos y estructuras que se dan también en otros medios de comunicación más complejos como el cine y TV, en su lenguaje, combina e integra diversos códigos: el verbal, mediante textos lingüísticos y el no verbal, con imágenes fijas y conversaciones específicas, influye en la creatividad del alumno, apoya para la comprensión de lo expuesto en clase, ayuda en la capacidad de síntesis y actúa como motivador en el campo de la lectura". Es decir, que por su diversidad de contenidos y diseños, han logrado combinar lo visual y lo verbal, razón por la cual existen catedráticos que han implementado la historieta en las aulas para un mejor aprendizaje.

Basándose en Eisner (2008) “el Manga es una narración gráfica, ya que posee el arte de crear imágenes estereotipadas con propósitos narrativos, describiendo imágenes en una historia, convirtiendo al Manga en un texto narrativo que se afirma en la imagen para un mejor entendimiento de la trama". Dicho en otras palabras, el Manga utiliza diferentes tipos de narraciones; por citar un ejemplo se menciona a la narración descriptiva en la cual una realidad específica se muestra con palabras indicando ambientes, personas, emociones y procesos. Además, no se pude descartar a la narración gráfica explicativa cuyo objetivo es demostrar una serie de etapas que conllevan a un final.

El Manga es un mundo amplio de historias divido en géneros y subgéneros. Este material está dirigido para todo público. Tomando en cuenta este antecedente se reconoce que es un recurso interesante para ser incluido en la educación, puesto que el docente podría utilizar el género que más se adapte a las necesidades educativas.

Entre los géneros adecuados al contexto de la presente investigación tenemos:

- Harem: es un género que basa su historia en un hombre que posee escasos talentos y pocos encantos. Sin embargo, el personaje tiene muchas chicas enamoradas de él, y durante la trama los personajes femeninos se pelean constantemente para intentar ganar el amor del protagonista. Los harems más conocidos son: Vampire, Love Hien, Tenchi muyo entre otros.

- Mecha: es un género futurista basado en gigantescos robots manejados por humanos. Entre los más populares se encuentran, Eureka 7, Tengen Toppa Gurren Lagann, La Visión de Escaflowne, etc. 
- Seinen: significa hombre joven y es un Manga dirigido a varones a partir de 18 años en adelante. Es un estilo contrario al Shonen, ya que las tramas son más fuertes en este género y se tratan temas como negocios y política. En este género, los personajes poseen poderes extraordinarios. Montecristo, Death note, Trinity blood y Cowboy son algunas series de esta índole.

- Josei: narra historias amorosas o de decepciones. El objetivo es llegar al público para que analice cómo esas experiencias pueden servir para ser mejores. Ejemplos de este género son: Paradise Kiss, Honey y Clover.

- Magical Girlfriend: en el Manga no hay fronteras así que en este género la trama se basa en la relación amorosa entre extraterrestres y humanos. Haruhi suzumiya pertenece a este estilo de Manga.

Quintana (2010), menciona que la comprensión es un proceso interactivo donde el lector va construyendo significados, siendo importante y oportuno analizar el constructivismo y sus estrategias. El constructivismo explica cómo el aprendiz unifica conocimientos, iniciando desde su experiencia hasta llegar a un verdadero aprendizaje significativo, es decir que será capaz no solo de receptar información sino de producir. Pensadores como Piaget, Vygotsky y Ausubel admiten que esta teoría permite al estudiante ser crítico, analítico y como ya se había mencionado creador de conocimiento.

Según Piaget (1980) el conocimiento es creado por el estudiante a partir de un conocimiento previo; es decir, él se encargada de reflexionar y experimentar para mejorar su instrucción.

Tabla 1. Cuadro comparativo Piaget y la Comprensión Lectora.

\begin{tabular}{|c|c|}
\hline Mecanismos según Piaget & Etapas de la comprensión lectora \\
\hline $\begin{array}{c}\text { Adquisición de conocimientos, a través } \\
\text { de una experiencia previa. }\end{array}$ & $\begin{array}{c}\bullet \text { Pre lectura } \\
\text { Presentación de contenidos para activar } \\
\text { el conocimiento. }\end{array}$ \\
$\begin{array}{c}\text { Acomodación } \\
\text { Transformación de información antigua a } \\
\text { nueva. }\end{array}$ & $\begin{array}{c}\text { Lectura } \\
\text { Identificación de ideas principales y } \\
\text { comprensión de significados. }\end{array}$ \\
\hline $\begin{array}{c}\text { Equilibrio } \\
\text { Aseveración de conocimientos. }\end{array}$ & $\begin{array}{c}\text { Post lectura } \\
\text { Aseveración de conocimientos. }\end{array}$ \\
\hline
\end{tabular}

Elaborado por: Vallejo Karina, 2018

La comprensión implica que cada lector percibe lo leído, por lo tanto, no se puede tener una interpretación objetiva en cada lector. Es por esto que los niveles de conocimiento lector ayudan al descifrador a determinar en qué nivel de comprensión se encuentra.

En los años 20, de acuerdo a la teoría conductista se consideraba que leer era verbalizar lo expuesto en el texto, prescindiendo de la 
interacción del lector con el escrito, y como consecuencia de ese sistema el lector no podía exponer sus ideas o nuevas hipótesis. En aquel tiempo, se estipulaba que leer era decodificar signos y sonidos en otras palabras, relacionar letras con fonemas; por ende, se pensaba que si una persona sabía identificar letras y sonidos sabía leer adecuadamente. Esto podría ser cierto, sin embargo, saber leer no es comprender ya que entender abarca mucho más que identificar sonidos y tener una buena pronunciación.

Por consiguiente, la lectura no solo es decodificar letras para posteriormente alcanzar la comprensión lectora, de hecho la lectura es un medio de comunicación a través del cual se puede entender lo desconocido o a su vez reafirmar conocimientos permitiendo al lector interactuar socialmente al momento de comprender el texto.

La comprensión lectora es la destreza receptiva de decodificación de un texto escrito que utiliza estrategias didácticas para empezar un proceso lector que implica: pre lectura, lectura y post lectura, con el fin de interpretar un contexto ya sea en un nivel literal, inferencial o crítico

La comprensión lectora está divida en tres niveles: literal, inferencial y crítico.

\section{Niveles de comprensión lectora}

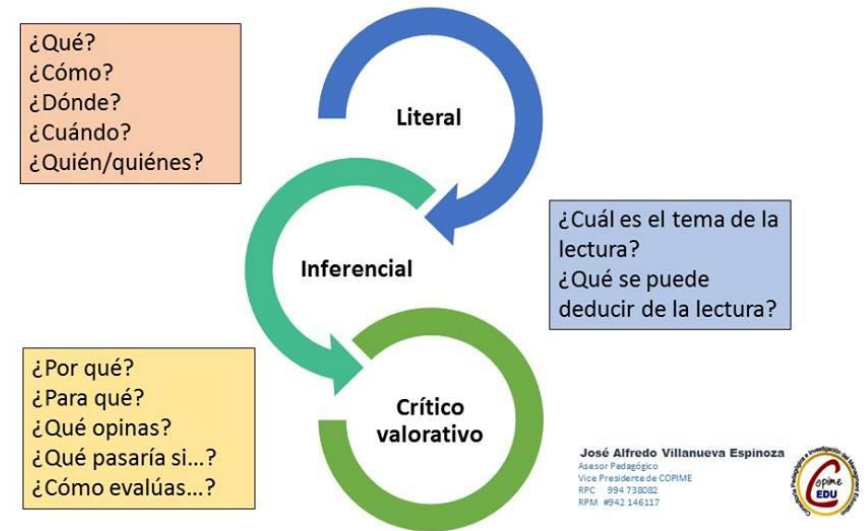

Gráfico 1. Imagen 5 Niveles de comprensión lectora Fuente: Perú educa

El lector decodifica textos, reconociendo expresiones y palabras específicas en un texto. Además, permite explorar conocimientos básicos, siendo este el nivel inicial para alcanzar una óptima comprensión. En este nivel el maestro debe ayudar a los estudiantes a:

- Identificar detalles.

- Reconocer tiempo, espacio y protagonistas de la historia.

- Entender la secuencia de los eventos relacionados al texto. 
- Comprender el significado de palabras, oraciones y fragmentos.

- Identificar sinónimos, antónimos.

La lectura crítica enmarca un sistema analítico, activo y reflexivo permitiendo al lector descubrir las ideas que resaltan dentro de un texto escrito para ser interpretadas lógicamente. Por tanto, comprender un texto en el nivel crítico significa juzgar la intención del autor plasmada en sus escritos; es decir, el lector debe establecer juicios valorativos basándose en argumentos que puedan sustentarse y que el lector pueda soportar de acuerdo a la información proporcionada por el autor.

Para Monroy (2014), para comprender un texto en este nivel el lector debe recurrir a su lógica y a todos los conocimientos adquiridos previamente referentes al texto o al tema del escrito, por ende las experiencias personales como lector serán de gran ayuda para las interpretaciones posteriores ya que todos estos elementos ayudarán al lector a adoptar una postura frente a lo que el autor manifiesta.

En este nivel existen juicios críticos que deben ser tomados en cuenta ya sea por su exactitud, aceptabilidad o probabilidad, siendo estos de realidad o fantasía, de adecuación y validez en donde el lector compara la información proporcionada con otras fuentes. Otro de los juicios es el de aprobación, el mismo que necesita ser evaluado relativamente en diferentes partes de la lectura para poder ser asimilado y por último el juicio de rechazo o aceptación en donde se pone en juego el código moral del lector.

El lector debe pasar por un proceso que facilite llegar a la comprensión lectora, por tanto, la lectura tiene etapas con actividades específicas que proporcionan pautas para que el conocimiento del lector

Para Sole (1992), la lectura se basa en subprocesos o etapas: en la primera etapa el lector identifica el contenido a ser estudiado mientras que en la segunda construye significados y en la etapa final utiliza mecanismos cognitivos para analizar, sintetizar y generalizar la información receptada del texto.

Teniendo en cuenta lo anteriormente expuesto, se entiende que la lectura es un proceso que permite la adquisición de destrezas cognitivas, afectivas y conductuales. Es necesario dividirla por etapas, las mismas que aplican como estrategias específicas en cada una. (Sole, 1992), clasifica la lectura en tres etapas: "Pre-lectura, lectura y Post-lectura". 


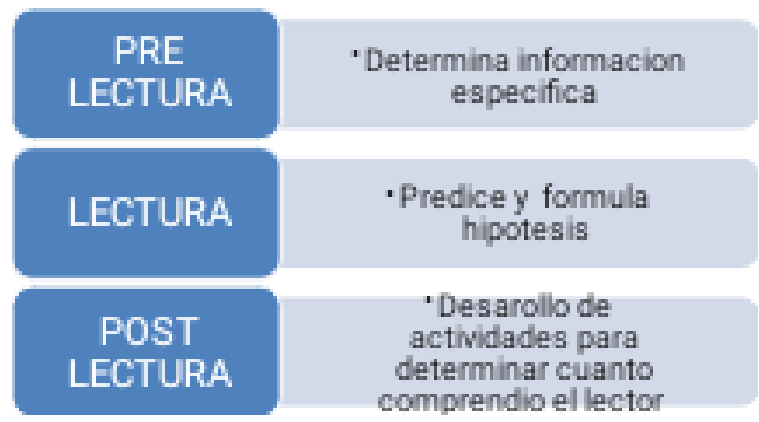

Gráfico 2. Etapas del proceso Lector Elaborado por: Vallejo Karina, 2018

\section{METODOLOGÍA}

La modalidad de la investigación fue socioeducativa, ya que estuvo dirigida a hacer un aporte a la educación a través de diferentes teorías y conocimientos metodológicos, los cuales buscan cambiar la educación tradicional, especialmente en lo que respecta a la comprensión lectora del idioma inglés.

La investigación tuvo un enfoque cuantitativo, ya que la información proviene de datos investigados, fuentes bibliográficas y además de resultados que fueron analizados estadísticamente a un examen previo y posterior, acerca de la comprensión lectora que fue aplicado a los estudiantes del Centro de Idiomas.

Se consideró un diseño cuasi-experimental, en base a una propuesta y un plan de intervención, que permitió observar el efecto de las dos variables en el contexto de la investigación. Es decir que, para mejorar la comprensión lectora del idioma inglés, los estudiantes se sujetaron a un plan de intervención, en el cual la variable independiente Manga tomó un rol de suma importancia para validar su incidencia sobre la comprensión lectora del idioma inglés.

La investigación también es correlacional, ya que se buscó la relación directa entre la estrategia Manga y la comprensión lectora del idioma inglés, incurriendo en los niveles exploratorio y descriptivo. Además, fue también una investigación de campo pues se realizó con los estudiantes de tercer nivel de inglés del Centro de Idiomas.

Está basada en información proveniente de investigaciones ya existentes (tipo bibliográfica), así como: conjeturas, hipótesis, experimentos, instrumentos y métodos ya usados en investigaciones anteriores con el fin de recabar información específica que ayude a dar solución al problema planteado.

La población está conformada por estudiantes del tercer nivel del Centro de Idiomas de la Escuela Superior Politécnica de Chimborazo, de los cuales se consideró una muestra intencional de 38 estudiantes entre hombres 
y mujeres; de los cuales 20 son del paralelo "1" (grupo de control) y 18 estudiantes son del paralelo "2" (grupo experimental) matriculados en el período académico septiembre 2018 - febrero 2019.

Tabla 2. Muestra.

\begin{tabular}{|c|c|c|c|}
\hline \multicolumn{4}{|c|}{ Grupos } \\
\hline Control & Número de Estudiantes & Experimental & Número de Estudiantes \\
\hline Tercero "1" & 20 & Tercero "2" & 18 \\
\hline Total muestra & \multicolumn{3}{|c|}{38 estudiantes. } \\
& \multicolumn{3}{|c|}{} \\
\hline
\end{tabular}

Fuente: Sistema académico

Elaborado por: Vallejo Karina, 2018

La variable independiente es Manga, que consiste en una historieta japonesa con dibujos en blanco y negro que se caracteriza por narrar historias reales, históricas o fantásticas en diferentes géneros. Su estilo es narrativo, los dibujos son originales, así como su lectura, ya que se leen de forma revertida y de arriba hacia abajo. Los textos e imágenes se encuentran en pequeñas viñetas que contienen lenguaje verbal y visual. (Vallejo, 2017); mientras que la variable dependiente considera la destreza receptiva de decodificación de un texto escrito, que utiliza estrategias didácticas para empezar un proceso lector que implica: pre lectura, lectura y post lectura, con el fin de interpretar un contexto ya sea en un nivel literal, inferencial o crítico (Vallejo, 2017).

La investigación se inicia sustentando la epistemología de las variables intervinientes que permitieron determinar los indicadores, indicadores que fueron evaluados mediante la elaboración de un cuestionario con base a una prueba estandarizada internacional de Cambridge de la sección de lectura (Reading section) el cual, al ser un instrumento de recolección de datos estandarizado, no necesitó validación.

Posteriormente se determinó que el grupo de control realice las actividades de forma tradicional; mientras que, para el grupo experimental, se desarrolló un plan de intervención que consistió en la aplicación de actividades utilizando manga como estrategia para incidir en la comprensión lectora.

De forma paralela, el grupo de control y experimental fueron evaluados a través del instrumento de recolección de datos en dos momentos: antes y después de aplicar el método tradicional y el plan de intervención (manga), lo que permitió en un inicio (pre test) diagnosticar la comprensión lectora de quienes representan la muestra de la investigación; y posteriormente (pre test) permitió evaluar el nivel de comprensión lectora en los estudiantes que realizaron las actividades tradicionales, así como quienes desarrollaron el plan de intervención (mangas). 
Los datos obtenidos por cada grupo fueron analizados por separado, para lo cual se utilizó el método teórico, de análisis, síntesis de procesamiento y sistematización de la información, Es decir, que la información fue tabulada y organizada, con el fin de que los resultados sean procesados en términos de medidas descriptivas como son: distribución de frecuencia, porcentajes, medias aritméticas, desviación típica y varianza.

Finalmente, mediante la prueba estadística $\mathrm{Z}$, se confrontó los datos obtenidos de los grupos de control y experimental, los mismos que permitieron comprobar el cumplimiento de la hipótesis planteada y poder determinar las conclusiones atinentes al contexto de la investigación.

\section{RESULTADOS}

\section{Análisis estadísticos de los Test aplicados a los estudiantes}

Después de aplicar los Test de evaluación se tabuló y organizó los resultados para ser procesados en términos de medidas descriptivas como son: distribución de frecuencia, porcentajes, medias aritméticas, desviación típica y varianza. pasos:

El proceso utilizado se describe a continuación en los siguientes

- En cada ítem, se determinó la calificación correspondiente según el nivel.

- Se organizó la información de los valores de las calificaciones obtenidas por los respectivos grupos de aplicación, en tablas de información.

- Se utilizó el programa Excel para procesar las tablas de información que resumen los valores obtenidos en los diferentes instrumentos de evaluación, determinando la frecuencia y luego los respectivos cálculos de frecuencia, media aritmética y desviación estándar.

- Se analizaron los datos obtenidos en términos descriptivos, con la finalidad de interpretarlos y responder a los objetivos de la investigación.

- Se confrontaron los hallazgos obtenidos con la teoría.

Para la prueba de hipótesis se eligió la prueba estadística de distribución normal $\mathrm{Z}$, que se denota con $\mathrm{Z}$ o simplemente $\mathrm{Z}$ al valor crítico que separa las áreas de rechazo y aceptación de la hipótesis nula. En un ensayo a dos colas, para un nivel de significación del 5\%, $\alpha=0,05$. 


\section{Pre - Test}

Tabla 3. Resultados Pre Test (Grupo Experimental Y Grupo de Control)

PRE Test - Grupo Experimental.

\begin{tabular}{|c|c|c|c|}
\hline Calificaciones & Frecuencias & \multirow{2}{*}{$\left(\mathrm{x}_{\mathrm{i}}\right)\left(\mathrm{f}_{\mathrm{i}}\right)$} & \multirow{2}{*}{$\left(\mathrm{x}_{\mathrm{i}}{ }^{2}\right)\left(\mathrm{f}_{\mathrm{i}}\right)$} \\
\cline { 1 - 2 }$\left(\mathrm{x}_{\mathrm{i}}\right)$ & $\left(\mathrm{f}_{\mathrm{i}}\right)$ & 0 & 0 \\
\hline 1 & 0 & 0 & 0 \\
\hline 2 & 0 & 0 & 0 \\
\hline 3 & 0 & 9 & 27 \\
\hline 4 & 3 & 24 & 96 \\
\hline 5 & 6 & 12 & 725 \\
\hline 6 & 5 & 14 & 98 \\
\hline 7 & 2 & 0 & 0 \\
\hline 8 & 2 & 0 & 0 \\
\hline 9 & 0 & $\sum \mathrm{x}_{\mathrm{i}} \cdot \mathrm{f}_{\mathrm{i}}=$ & $\sum \mathrm{x}_{\mathrm{i}}^{2} \mathrm{f}_{\mathrm{i}}=$ \\
\hline 10 & 0 & 84 & 418 \\
\hline
\end{tabular}

PRE Test - Grupo Control

\begin{tabular}{|c|c|c|c|}
\hline Calificaciones & Frecuencias & \multirow{2}{*}{$\left(\mathrm{x}_{\mathrm{i}}\right)\left(\mathrm{f}_{\mathrm{i}}\right)$} & \multirow{2}{*}{$\left(\mathrm{x}_{\mathrm{i}}^{2}\right)\left(\mathrm{f}_{\mathrm{i}}\right)$} \\
\hline$\left(\mathrm{x}_{\mathrm{i}}\right)$ & $\left(f_{i}\right)$ & & \\
\hline 1 & 0 & 0 & 0 \\
\hline 2 & 0 & 0 & 0 \\
\hline 3 & 2 & 4 & 8 \\
\hline 4 & 2 & 6 & 18 \\
\hline 5 & 5 & 20 & 80 \\
\hline 6 & 5 & 25 & 125 \\
\hline 7 & 3 & 18 & 108 \\
\hline 8 & 1 & 7 & 49 \\
\hline 9 & 2 & 16 & 128 \\
\hline 10 & 0 & 0 & 0 \\
\hline & $\Sigma \mathrm{fi}=20$ & $\begin{array}{c}\Sigma \times \text { xi. fi }= \\
96\end{array}$ & $\begin{array}{l}\Sigma \times i 2 \mathrm{fi} \\
=516\end{array}$ \\
\hline
\end{tabular}

Fuente: Pre Test (Grupo Experimental y Grupo de Control)

Elaborado por: la investigadora

\section{Para el análisis se toma en cuenta la siguiente nomenclatura:}

$\sigma: \quad$ Desviación típica.

$\Sigma$ f: Sumatoria de las frecuencias

$\mathrm{N}$ : Número total de casos.
$\Sigma \mathrm{x}$ : Variables (calificaciones).

n: Número total de datos.

\section{Cálculo para los grupos de aplicación, instrumento: Pre Test}

\section{Cálculo de la media aritmética:}

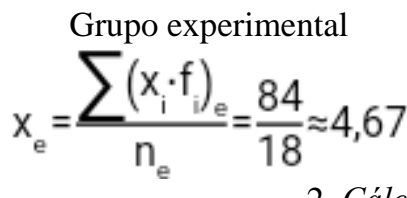

2. $C$

Grupo experimental

$$
\begin{aligned}
& \sigma_{\mathrm{e}}=\sqrt{\frac{\sum \mathrm{x}_{\mathrm{i}}^{2} \mathrm{f}_{\mathrm{i}}}{\mathrm{n}_{\mathrm{e}}}-\mathrm{x}_{\mathrm{e}}} \\
& \sigma_{e}=\sqrt{\frac{418}{18}-4,67^{2}}
\end{aligned}
$$$$
\sigma_{\mathrm{e}}=1,20
$$

$$
\mathrm{x}_{\mathrm{c}}=\frac{\sum\left(\mathrm{x}_{\mathrm{i}} \cdot \mathrm{f}_{\mathrm{i}}\right)_{\mathrm{c}}}{\mathrm{n}_{\mathrm{c}}}=\frac{96}{20} \approx 4,80
$$

Grupo de control

$$
\begin{gathered}
\sigma_{\mathrm{c}}=\sqrt{\frac{\sum \mathrm{x}_{\mathrm{i}}^{2} \mathrm{f}_{\mathrm{i}} \mathrm{n}_{\mathrm{c}}{ }^{2}}{\mathrm{x}_{\mathrm{c}}}} \\
\sigma_{\mathrm{c}}=\sqrt{\frac{516}{20}-4,8^{2}}
\end{gathered}
$$

$\sigma_{c}=1,66$ 


\section{PRE TEST}

4.90

$4.85 \longrightarrow$ 4.30

4.80

4.75

4.70

4.65

4.60

4.55

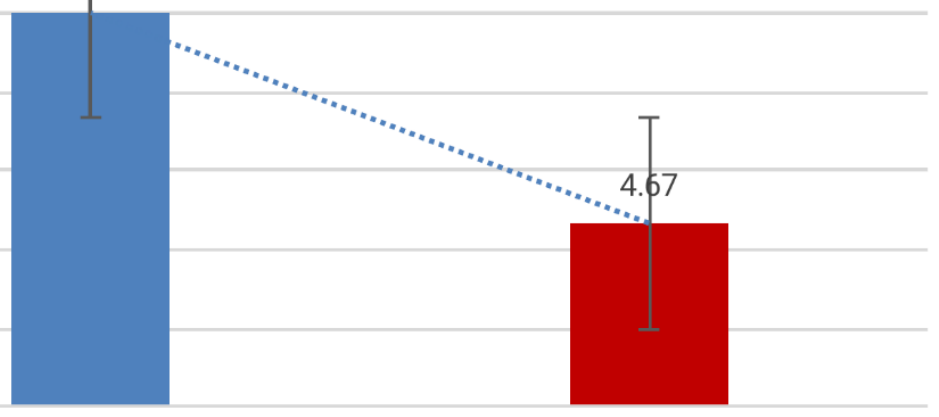

PRE - CONTROL

PRE EXPERIMENTAL

Gráfico 1. Pre Test en los grupos de aplicación.

El promedio que obtuvo el grupo experimental es de 4,67/10 el cual corresponde al 46,7\%, y el grupo de control obtuvo como promedio 4,8/10 correspondiente al $48 \%$, se observa que la diferencia es de 0,13 correspondiente al $1,3 \%$. Por lo tanto, se concluye que los grupos experimental y de control disponen de los prerrequisitos necesarios para comenzar el estudio.

\section{Post Test}

Tabla 4. Resultados post - Test (Grupo Experimental y Grupo de Control)

Grupo Experimental

\begin{tabular}{|c|c|c|c|}
\hline Calificaciones & Frecuencias & \multirow{2}{*}{$\left(\mathrm{x}_{\mathrm{i}}\right)\left(\mathrm{f}_{\mathrm{i}}\right)$} & \multirow{2}{*}{$\left(\mathrm{x}_{\mathrm{i}}{ }^{2}\right)\left(\mathrm{f}_{\mathrm{i}}\right)$} \\
\hline$y^{\left(\mathrm{x}_{\mathrm{i}}\right)}$ & $\left(\mathrm{f}_{\mathrm{i}}\right)$ & 0 & 0 \\
\hline 1 & 0 & 0 & 0 \\
\hline 2 & 0 & 0 & 0 \\
\hline 3 & 0 & 0 & 0 \\
\hline 4 & 0 & 4 & 16 \\
\hline 5 & 1 & 5 & 25 \\
\hline 6 & 1 & 24 & 144 \\
\hline 7 & 4 & 28 & 196 \\
\hline 8 & 4 & 40 & 320 \\
\hline 9 & 5 & 18 & 162 \\
\hline 10 & 2 & & $\Sigma \mathrm{x}_{\mathrm{i}}{ }^{2} \mathrm{f}_{\mathrm{i}}=$ \\
\hline & & $\sum \mathrm{x}_{\mathrm{i}} . \mathrm{f}_{\mathrm{i}}=196$ & 936 \\
\hline
\end{tabular}

\begin{tabular}{|c|c|c|c|}
\hline & & & \\
\hline Calificaciones & Frecuencias & (x.)(f.) & $\left(x^{2}\right)(f)$ \\
\hline$\left(\mathrm{x}_{\mathrm{i}}\right)$ & $\left(f_{i}\right)$ & $\left(\mathrm{i}_{\mathrm{i}}\right)\left(\mathrm{I}_{\mathrm{i}}\right)$ & $\left(x_{i}\right)\left(I_{i}\right)$ \\
\hline 1 & 0 & 0 & 0 \\
\hline 2 & 0 & 0 & 0 \\
\hline 3 & 0 & 0 & 0 \\
\hline 4 & 3 & 9 & 27 \\
\hline 5 & 2 & 8 & 32 \\
\hline 6 & 6 & 30 & 150 \\
\hline 7 & 5 & 30 & 180 \\
\hline 8 & 3 & 21 & 147 \\
\hline 9 & 1 & 8 & 64 \\
\hline 10 & 0 & 0 & 0 \\
\hline & $\Sigma \mathrm{f}_{\mathrm{i}}=20$ & $\sum \mathrm{x}_{\mathrm{i}} \cdot \mathrm{f}_{\mathrm{i}}=106$ & $\begin{array}{l}\Sigma \mathrm{x}_{\mathrm{i}}^{2} \mathrm{f}_{\mathrm{i}} \\
=600\end{array}$ \\
\hline
\end{tabular}


Cálculo para los grupos de aplicación, instrumento: Post Test

1. Cálculo de la media aritmética:

$\mathrm{x}_{\mathrm{e}}=\frac{\sum\left(\mathrm{x}_{\mathrm{i}} \cdot \mathrm{f}_{\mathrm{i}}\right)_{\mathrm{e}}}{\mathrm{n}_{\mathrm{e}}}=\frac{196}{18} \approx 7,17$

$\mathrm{x}_{\mathrm{c}}=\frac{\sum\left(\mathrm{x}_{\mathrm{i}} \cdot \mathrm{f}_{\mathrm{i}}\right)_{\mathrm{c}}}{\mathrm{n}_{\mathrm{c}}}=\frac{106}{20} \approx 5,30$

2. Cálculo de la desviación típica:

Grupo experimental

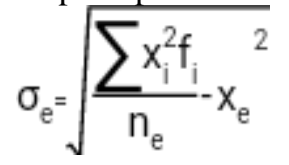

Grupo de control

$\sigma_{e}=\sqrt{\frac{936}{18}-7,17^{2}}$

$\sigma_{c}=\sqrt{\frac{\sum x_{i}^{2} f_{i}}{n_{c}}-x_{c}}$

$\sigma_{e}=1,46$

$\sigma_{c}=\sqrt{\frac{600}{20}-5,30^{2}}$

$\sigma_{\mathrm{c}}=1,48$

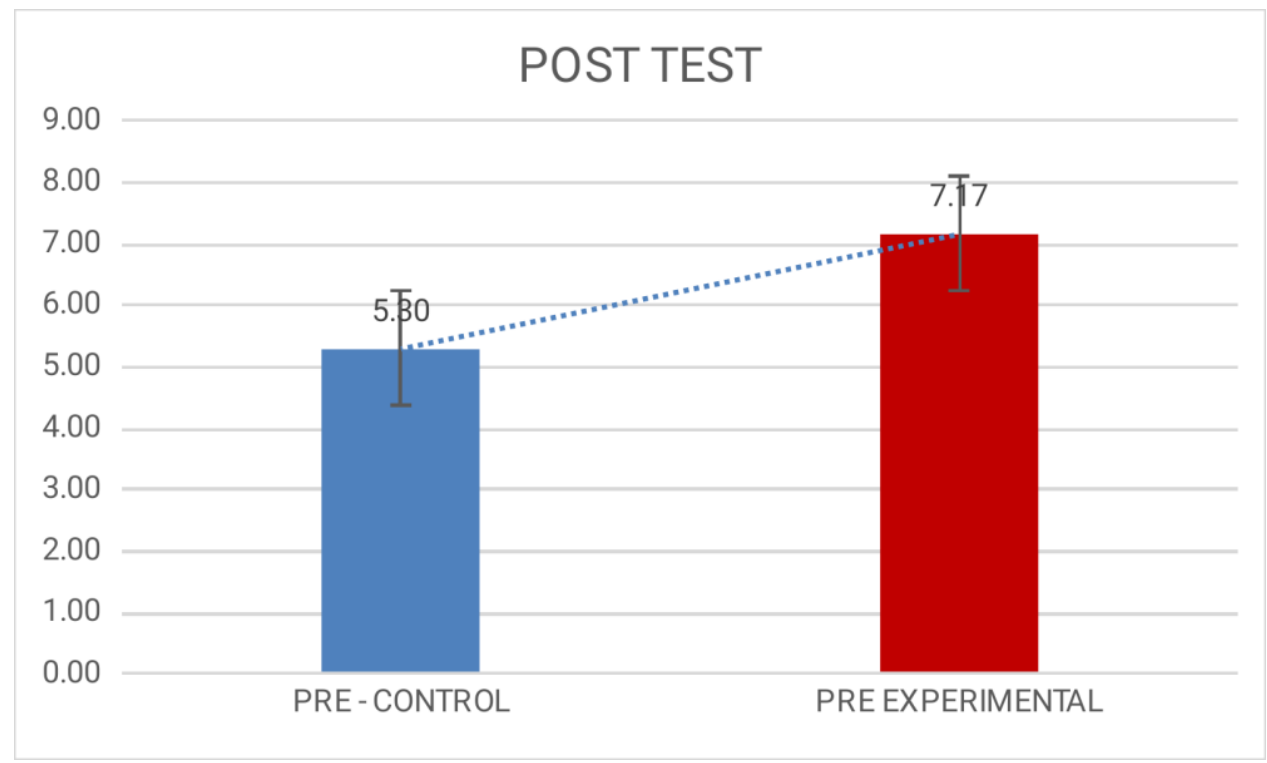

Gráfico 2. Post Test en los grupos de aplicación.

El promedio que obtuvo el grupo experimental es de 7,17/10 el cual corresponde al 71.7\%, y el grupo de control obtuvo como promedio 5.30/10 correspondiente al 53\%. Se observa que el grupo experimental con el cual se aplica la estrategia metodológica Manga obtuvo mejores resultados en el desarrollo de la comprensión lectora en el idioma inglés. 


\section{Análisis y Prueba de Hipótesis General}

Hi: La lectura de Manga influye en la comprensión lectora del idioma ingles de los estudiantes de tercer nivel del Centro de Idiomas de la Escuela Superior Politécnica de Chimborazo durante el periodo académico septiembre 2018 - febrero 2019.

Ho: El Manga no influye en la comprensión lectora del idioma inglés de los estudiantes de tercer nivel del Centro de Idiomas, de la Escuela Superior Politécnica de Chimborazo durante el periodo académico septiembre 2018 - febrero 2019.

Tabla5: Registro de los valores estadísticos obtenidos por los grupos de aplicación

\begin{tabular}{|c|c|c|c|c|}
\hline \multirow{2}{*}{ Test } & \multicolumn{2}{|c|}{ Grupo Experimental } & \multicolumn{2}{c|}{ Grupo Control } \\
\cline { 2 - 5 } & Media & $\begin{array}{c}\text { Desviación } \\
\text { Estándar ( } \boldsymbol{\sigma})\end{array}$ & $\begin{array}{c}\text { Media } \\
\text { Aritmética ( X } \\
\text { Aritmética ( X ) }\end{array}$ & $\begin{array}{c}\text { Desviación } \\
\text { Estándar }(\boldsymbol{\sigma})\end{array}$ \\
\hline Pre - Test & 4,67 & 1.20 & 4,8 & 1,66 \\
\hline Post - Test & 7,17 & 1,46 & 5,30 & 1,38 \\
\hline Promedio & 5,92 & 1,46 & 5,05 & 1,52 \\
\hline
\end{tabular}

Fuente: Pre-Test y Post-Test

Elaborado por: Vallejo Karina, 2018.

Para establecer las regiones de rechazo se utilizaron los criterios de niveles de confianza definidos por la siguiente ecuación para un intervalo de $95 \%$ de aceptación, por lo que el Nivel de confianza $=(1-\alpha) * 100 \%$; donde el nivel de significancia “ $\alpha$ ” será igual al 5\%

$$
\text { Por lo que: } a=\frac{5 \%}{2} ; a=2,5 \%
$$

El intervalo de confianza está en una distribución normal; la confianza se dividió para dos. Este valor corresponde a la simetría de cola superior e inferior de dicho repartimiento.

Para el nivel de confianza del 0,95 (95\%) cociente entre dos, obtendremos un valor resultante de 0,475 . Este valor pertenece en la tabla de distribución normal de probabilidades a un número $Z=1,96$ equivalente al $2,5 \%$, valor teórico proporcionado a las zonas de rechazo de una distribución normal Z.

\section{Cálculos con la prueba paramétrica $Z$}

Para el siguiente análisis se toma en cuenta la presente nomenclatura:

$x_{e}$ :

Media aritmética del grupo experimental.

$x_{\mathrm{e}}$ :

Media aritmética del grupo de control.

$\sigma_{\mathrm{e}}: \quad$ Desviación típica del grupo experimental. 
$\sigma_{\mathrm{c}}: \quad$ Desviación típica del grupo de control.

$\mathrm{n}_{\mathrm{e}}$ : Número de estudiantes del grupo experimental.

$\mathrm{n}_{\mathrm{e}}$ : Número de estudiantes del grupo de control.

Los datos obtenidos de la investigación son:

$$
\begin{array}{lll}
x_{e}=5,92 & & x_{c}=5,05 \\
\sigma_{e}=1,46 & & \sigma_{c}=1,52 \\
& n_{e}=18 & n_{c}=20
\end{array}
$$

Reemplazando los datos en la fórmula del puntaje $\mathrm{z}$ :

$$
\begin{gathered}
Z_{c}=\frac{x_{e}-x_{c}}{\sqrt{\frac{\sigma_{e}^{2}}{n_{e}}+\frac{\sigma_{c}^{2}}{n_{c}}}} \\
Z c \approx \frac{5,92-5,05}{\sqrt{\frac{(1,46)^{2}}{18}+\frac{(1,52)^{2}}{20}}} \\
Z_{c} \approx 3,24
\end{gathered}
$$

\section{Toma de decisión estadística}

Comparando los valores: Zc (calculado) y Zt (teórico), tenemos que:

$$
\begin{gathered}
Z_{c}=3,24 ; Z_{t}=1,96 \\
3,24>1,96 \\
Z_{c}>Z_{t}
\end{gathered}
$$

Lo cual conlleva a rechazar la hipótesis nula $\mathrm{Ho} \mathrm{x}_{\mathrm{e}}=\mathrm{x}_{\mathrm{C}} \mathrm{y}$ simultáneamente a aceptar la hipótesis de investigación Hi: $\mathrm{X}_{\mathrm{e}^{\neq}} \mathrm{X}_{\mathrm{C}}$ con la alternativa A1: $\mathrm{X}_{\mathrm{e}}>\mathrm{X}_{\mathrm{C}}$, es decir:

Hi: La lectura de Manga influye en la comprensión lectora del idioma inglés en los estudiantes de tercer nivel del Centro de Idiomas, de la Escuela Superior Politécnica de Chimborazo durante el periodo académico septiembre 2018 -febrero 2019.

El índice en el desarrollo de la comprensión lectora obtenido por los estudiantes de tercer nivel del Centro de Idiomas, de la Escuela Superior Politécnica de Chimborazo durante el periodo académico septiembre 2018 febrero 2019, periodo en el cual desarrollaron la temática del idioma Inglés con el uso de la estrategia metodológica Manga (Grupo experimental) supera 
al índice obtenido por los estudiantes que no utilizaron ésta estrategia en el desarrollo de la temática mencionada (Grupo control).

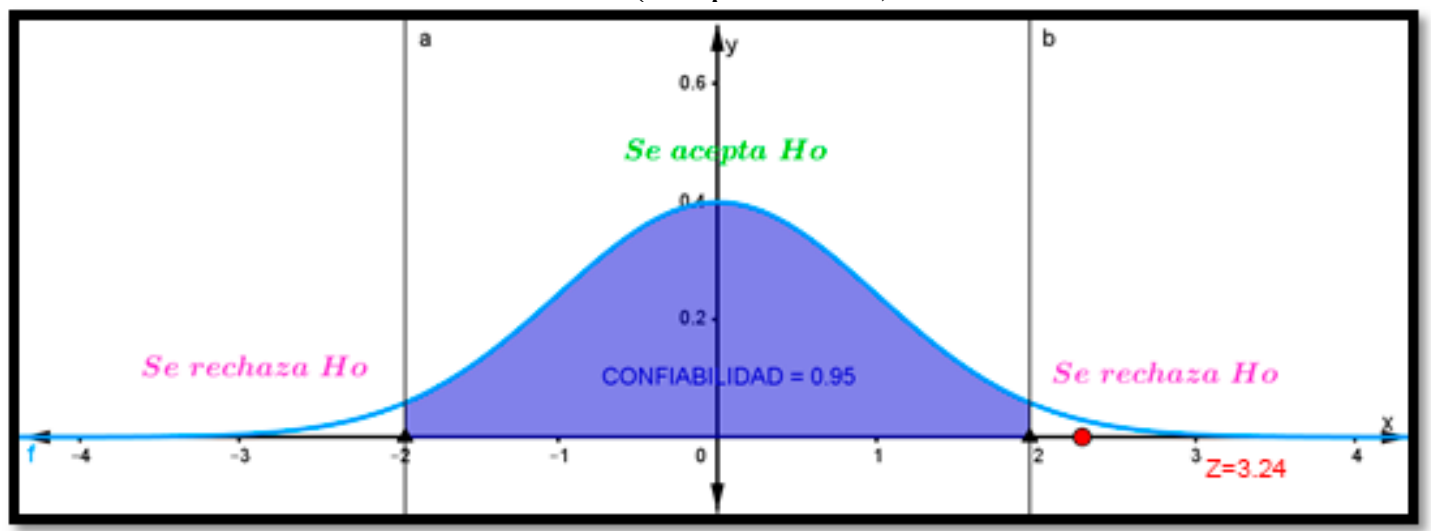

Gráfico 3. Interpretación Gráfica de los valores de la $\mathrm{Z}$ teórico y la $\mathrm{Z}$ calculado.

\section{DISCUSIÓN}

De los resultados obtenidos en la investigación, se determinó que el uso del Manga si mejora la comprensión lectora, pues tanto la investigación teórica como estadística así lo demuestran. Por otro lado, se comprobó que los estudiantes mejoraron su nivel lector a través de las actividades planteadas en la propuesta, ya que fueron aplicadas adecuadamente.

El Manga al ser utilizado por el docente como un recurso didáctico, permitió incidir en la compresión lectora a través de la pre lectura, lectura y post lectura. Retomando las palabras de (Garrido, 2010) “ La historieta contiene cualidades lingüisticas que permiten alcanzar un aprendizaje significativo debido a los procesos y técnicas que este posee". Con este antecedente y los resultados estadísticos que demostraron que el grupo experimental tuvo una media aritmética de 4,67 al inicio de la investigación y de 7.17 después de la aplicación de la propuesta, se determina que los estudiantes mejoraron la compresión lectora y su aprendizaje fue más significativo.

En contraparte, el grupo control obtuvo una medida aritmética de 4,8 en el pre test y 5,30 en el post test, lo que demuestra que los estudiantes no obtuvieron una mejora significativa ya que en este grupo no se aplicó el Manga como recurso para mejorar su comprensión lectora.

El cálculo del puntaje $\mathrm{Z}$ arroja un valor de 3.24, ubicándose en la zona de rechazo de la Hipótesis nula; es decir, se encuentra en la zona aceptación de la hipótesis de investigación.

Por lo anterior, se acepta la hipótesis planteada y se concluye que el Manga si influye en la comprensión lectora del idioma inglés en los estudiantes del contexto de la presente investigación. 
Cabe destacar que la técnica complementaria aplicada y el instrumento utilizado arrojaron datos significativos para esta investigación. Es así que se determina que el $87 \%$ de la población mejoró el nivel de comprensión lectora a través de la lectura de Mangas, lo cual quiere decir que este recurso didáctico evidentemente hace un aporte al mejoramiento de la destreza respectiva ya que el lenguaje tanto verbal como visual facilita la comprensión lectora.

\section{CONCLUSION}

Con base en los valores obtenidos del puntaje Z, se aceptó la hipótesis alterna, la cual sostiene que el Manga influye positivamente en el desarrollo de la comprensión lectora del grupo experimental de los estudiantes del tercer nivel del Centro de Idiomas, de la Escuela Superior Politécnica de Chimborazo durante el periodo académico septiembre 2018 febrero 2019. Esto quiere decir que existe una influencia de la variable independiente sobre la variable dependiente.

Previo a la implementación del Manga (historietas japonesas), el nivel de compresión lectora de los estudiantes de tercer nivel del Centro de Idiomas de la ESPOCH, era bajo (grupo experimental y grupo control), hecho evidenciado en los resultados del pre test con un puntaje promedio de 4.73 sobre 10. Sin embargo, la implementación de Manga influyó positivamente en los estudiantes del grupo experimental, permitiendo no sólo mejorar la comprensión lectora sino también la adquisición de vocabulario y la creatividad.

Para mejorar la compresión lectora del idioma inglés es necesario utilizar recursos innovadores que activen el interés por la lectura. En este sentido, la utilización de Mangas es un recurso didáctico acertado e innovador que activó el interés de la lectura y permitió a los estudiantes optimizar su desenvolvimiento, adquirir un nuevo hábito lector, mejorar su vocabulario y por ende perfeccionar la destreza receptiva ya que se contó con estrategias cognitivas, meta cognitivas, motivadoras y contextuales.

Aplicado el tratamiento al grupo experimental, el puntaje promedio de los estudiantes fue de 6,23 sobre 10, superando el puntaje de 4.7 obtenido en el pre test. De la misma manera, cabe señalar que el puntaje del grupo control en el post test, al no haber sido parte del cuasi experimento, se mantuvo en 4,8, lo cual demuestra un cambio significativo en los estudiantes que fueron parte del plan de intervención. Por lo que se debe considerar sistematizar procesos de evaluación de los aprendizajes, poniendo énfasis en los diagnósticos, ya que proporcionan datos fundamentales para iniciar actividades de enseñanza-aprendizaje basados en realidades de los estudiantes y de esta manera no desperdiciar recursos favorables dentro del aula. 


\section{References:}

1. Abal Alonso (2010). Marina, «El cómic en la clase de ELE: Una propuesta didáctica», Suplementos Marco ELE, disponible en http://marcoele.com/suplementos/el-comic-en-ele/

2. Basensi (14 de 2 de 2010). Aprende con los Comics. Obtenido de https://comic4.wordpress.com/el-comi-y-educacion/

3. Castillo, R. M. (2013). Otra educación. Obtenido de http://otraeducacion.blogspot.com/2014/12/ecuador-lector.html

4. Censos, I. N. (28 de noviembre de 2012). INEC. Obtenido de http://www.ecuadorencifras.gob.ec/3-de-cada-10-ecuatorianos-nodestinan-tiempo-a-la-lectura/

5. Eisner (2008).

6. Entre libros \& Letras. (11 de septiembre de 2014). Obtenido de iberolibro.com:

https://www.iberlibro.com/blog/index.php/2014/09/11/el-origen-dela-historieta-o-comic-y-su-evolucion/

7. Flores, R. (Octubre de 2010). Humor y educación. Obtenido de http://elhumorenlaeducacion.blogspot.com/2010/10/pedagogia-delhumor.html

8. Garrido, R. (2010). Guia del Comic. En R. Garrido, Don Pablito (págs. 70-79).

9. Monroy, V. D. (2014). Comprensión Lectora . En V. d. Monroy, Comprensión Lectora . Digital UNID.

10. Ortega, N. P. (1998). El problema de la comprensión lectora. Correo del Maestro, 7-8.

11. Página oficial de los Salones del Cómic y el Manga que se celebran en Barcelona. Federació d'Institucions Professionals del Còmic (11 de Abril de 2016).

12. Piaget (1980). Mindstorms: Children, Computers and Powerful Ideas. NEW YORK: Basic Books.

13. Sole, I. (1992). Estrategias de lectura. Barcelona: Grao.

14. Vega, C. F. (2012). El Cómic en el Ecuador y una propuesta de Cómic. Cuenca. 